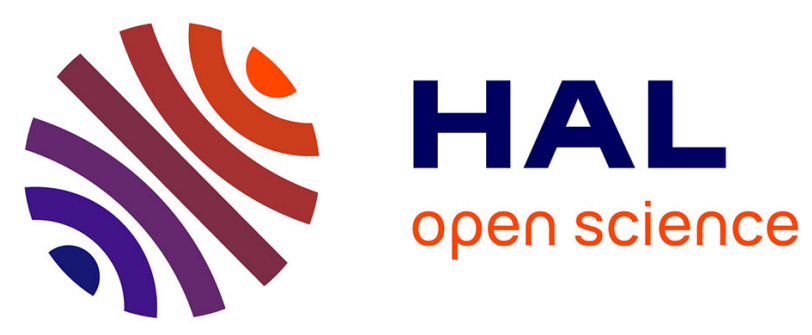

\title{
Direct Evidence of Flexomagnetoelectric Effect Revealed by Single-Molecule Spectroscopy
}

Ivan S. Veshchunov, Sergei V. Mironov, William Magrini, Vasili S. Stolyarov, A.N. Rossolenko, V.A. Skidanov, Jean-Baptiste Trebbia, Alexandre I. Buzdin, Philippe Tamarat, Brahim Lounis

\section{To cite this version:}

Ivan S. Veshchunov, Sergei V. Mironov, William Magrini, Vasili S. Stolyarov, A.N. Rossolenko, et al.. Direct Evidence of Flexomagnetoelectric Effect Revealed by Single-Molecule Spectroscopy. Physical Review Letters, 2015, 115 (2), pp.027601 (1-5). 10.1103/PhysRevLett.115.027601 . hal-01182344

\section{HAL Id: hal-01182344 \\ https://hal.science/hal-01182344}

Submitted on 31 Jul 2015

HAL is a multi-disciplinary open access archive for the deposit and dissemination of scientific research documents, whether they are published or not. The documents may come from teaching and research institutions in France or abroad, or from public or private research centers.
L'archive ouverte pluridisciplinaire HAL, est destinée au dépôt et à la diffusion de documents scientifiques de niveau recherche, publiés ou non, émanant des établissements d'enseignement et de recherche français ou étrangers, des laboratoires publics ou privés. 


\title{
Direct Evidence of Flexomagnetoelectric Effect Revealed by Single-Molecule Spectroscopy
}

\author{
I. S. Veshchunov, ${ }^{1,2}$ S. V. Mironov, ${ }^{3}$ W. Magrini, ${ }^{1,2,3}$ V. S. Stolyarov, ${ }^{4}$ A. N. Rossolenko, ${ }^{4}$ V. A. Skidanov, ${ }^{5}$ \\ J.-B. Trebbia, ${ }^{1,2}$ A. I. Buzdin, ${ }^{3}$ Ph. Tamarat, ${ }^{1,2}$ and B. Lounis ${ }^{1,2}$ \\ ${ }^{1}$ University Bordeaux, LP2N, F 33405 Talence, France \\ ${ }^{2}$ Institut d'Optique and CNRS, LP2N, F 33405 Talence, France \\ ${ }^{3}$ University Bordeaux, LOMA, F 33405 Talence, France \\ ${ }^{4}$ Institute of Solid State Physics, Russian Academy of Sciences, 142432 Chernogolovka, Russia \\ ${ }^{5}$ Institute for Design Problems in Microelectronics, Russian Academy of Sciences, 124681 Moscow, Russia
}

(Received 18 February 2015; published 6 July 2015)

\begin{abstract}
We report direct evidence of the electric field induced by a magnetization inhomogeneity in an iron garnet film. This inhomogeneity was created by the nonuniform magnetic fields generated at domain boundaries of a type I superconductor in the intermediate state. At liquid helium temperatures, Stark shifts of sharp single molecule zero phonon lines were used to probe the local electric fields generated by this flexomagnetoelectric effect. The measured electric fields are in accordance with theoretical estimations.
\end{abstract}

PACS numbers: 77.55.Nv, 32.60.+i, 33.70.Jg, 75.70.Kw

The interplay between magnetism and electricity in multiferroic materials attracts growing theoretical and experimental interests [1,2]. These materials offer the possibility of controlling the magnetization without applying electric currents, opening the way for the development of new nanoscale memory elements with low power consumption [2,3]. An electric field applied to such materials can produce substantial changes in the magnetic moment distribution. This magnetoelectric effect, which is due to the Dzyaloshinskii-Moriya interaction [4], has been revealed by Faraday rotation of light polarization in a ferrite garnet crystal under an external electric field [5,6]. Conversely, an inhomogeneous magnetization can induce an electric polarization in systems with broken inversion symmetry. This so-called flexomagnetoelectric effect [7] was recently predicted for spiral multiferroics where the geometrical magnetic frustration results in periodically modulated magnetization [8,9]. Even in centrosymmetric magnetic materials, a local violation of the inversion symmetry in cycloid magnetic defects (such as Néel domain walls) can give rise to the "flexomagnetoelectric" effect [10]. Moreover, the spatial configuration of the induced electric field in these materials can easily be controlled by the manipulation of the domain walls [11].

The electric polarization $\mathbf{P}(\mathbf{r})$ induced by the nonuniform magnetization $\mathbf{M}(\mathbf{r})$ has the form [12-15]

$$
\mathbf{P}=\gamma \chi_{e}[(\mathbf{M} \cdot \nabla) \mathbf{M}-\mathbf{M}(\nabla \cdot \mathbf{M})],
$$

where $\gamma$ is the magnetoelectric coupling constant and $\chi_{e}$ is electric susceptibility. According to this formula, Néel walls with in-plane rotation of the magnetization vector can be the source of a spontaneous nonuniform electric polarization, while Bloch walls are electrically uncharged $[1,4]$. Although the flexomagnetoelectric effect for the Néel-type domain walls was theoretically predicted more than 20 years ago [10], its unambiguous experimental evidence is still lacking. The manipulation of Néel domain walls by external electric fields has been considered as an indirect proof of the flexomagnetoelectric nature of the induced electric polarization in rare-earth iron garnets $[13,14]$. However, the interpretation of these experimental results remains controversial. The manipulation can indeed result from other mechanisms such as the strong electric field gradients generated in the vicinity of a needlelike electrode, which may induce changes in the magnetization distribution [16]. In this Letter, we provide direct experimental evidence of the electric polarization induced by the magnetization inhomogeneity in Bismuth substituted lutetium iron garnet (Bi:LuIG) films, using singlemolecule spectroscopy. The strong inhomogeneous magnetization is produced by stray magnetic fields in the vicinity of a type-I superconductor in the intermediate state.

Single aromatic molecules trapped in well-chosen matrices at liquid helium temperatures display a sharp zerophonon optical resonance with a quality factor up to $\sim 10^{8}$ (resonances in the visible spectrum with a linewidth of a few tens of megahertz) $[17,18]$. Centrosymmetric aromatic molecules embedded in disordered solid matrices usually gain permanent dipole moments due to distortions by the surrounding matrix. As a result, their lowest singlet-singlet electronic transition undergoes a linear Stark shift up to $\sim 1 \mathrm{GHz} /(\mathrm{MV} / \mathrm{m})[19,20]$. This transition is almost insensitive to magnetic fields since their diamagnetic redshift is lower than $10 \mathrm{MHz} / \mathrm{T}^{2}$ [21]. Single molecules are thus appropriate ultrasensitive probes of local electric fields induced by an inhomogeneous magnetization in Bi:LuIG.

The sample was composed of a superconducting indium layer (thickness $D=1.6 \mu \mathrm{m}$ ) grown by thermal evaporation on a silicon substrate, a molecular film of hexadecane (thickness $d \sim 0.1 \mu \mathrm{m}$ ) doped with fluorescent 

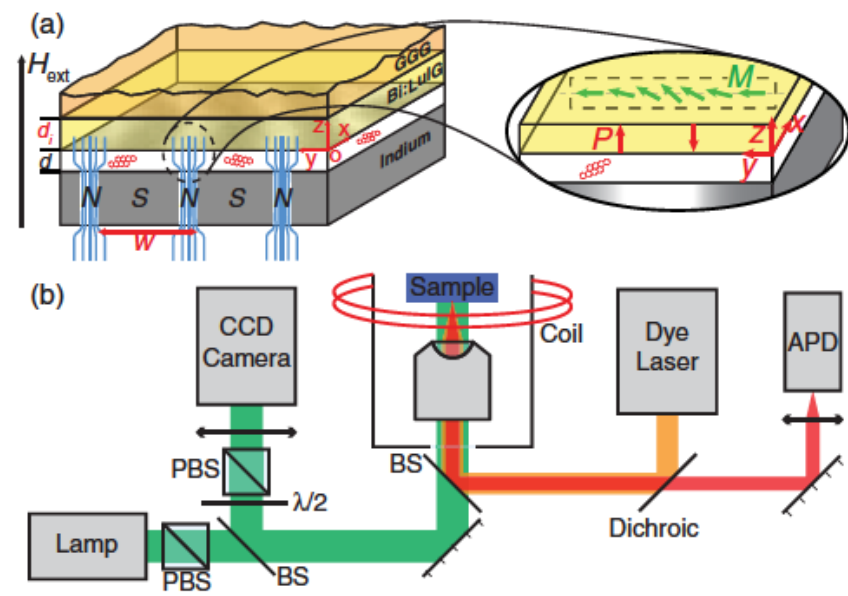

FIG. 1 (color online). Scheme of the sample under an external magnetic field. A hexadecane layer doped with dibenzanthan threne molecules is squeezed between an indium film and a $\mathrm{Bi}: \mathrm{LuIG}$ layer grown on a GGG substrate. (Inset) Scheme of magnetization distribution in the $\mathrm{Bi}$ : LuIG layer close to a normal phase domain of indium. Electric polarization vectors near the neighboring domain walls have opposite directions. (b) Scheme of the optical setup for single molecule microspec troscopy and simultaneous MO imaging of the regions where the vertical component of the magnetic field penetrates the $\mathrm{Bi}$ :LuIG layer.

dibenzanthanthrene molecules [22] at a concentration $\sim 10{ }^{6} \mathrm{M}$, and a Bi:LuIG layer (thickness $d_{i}=2.5 \mu \mathrm{m}$ ) with in-plane uniform saturation magnetization $M_{s}=50 \mathrm{G}$, grown on a $500 \mu \mathrm{m}$ thick gadolinium gallium gamet (GGG) substrate by liquid phase epitaxy [See Fig. 1(a)]. It was mounted on a piezo scanner and inserted together with a microscope objective (numerical aperture 0.5 ) in a helium cryostat. The sample was cooled below the critical temperature of indium $\left(T_{c}=3.4 \mathrm{~K}\right)$ and then submitted to an external magnetic field $\mathbf{H}_{\text {ext }}=H_{\text {ext }} \hat{\mathbf{z}}$ in order to set the indium film in the superconducting intermediate state. The optical microscopy setup is depicted in Fig. 1(b) [23]. It allows magneto-optical (MO) imaging of the regions where the vertical component of the magnetic field penetrates the $\mathrm{Bi}$ :LuIG layer, i.e., the regions close to the normal-phase domains of the indium film. MO imaging is indeed based on the Faraday rotation of light polarization in the Bi: LuIG indicator [33-36], in a crossed-polarizer beam path configuration, and CCD camera imaging [37,38]. This setup is also used to perform single-molecule spectroscopy. The fluorescent molecules are excited on their zero-phonon singlet-singlet transition with a tunable single frequency dye laser (589 nm, spectral width $1 \mathrm{MHz}$ ). Their redshifted fluorescence on vibrational transitions was focused either on the CCD camera for wide-field imaging or onto an avalanche photodiode for confocal microscopy.

Figure 2(a) shows a MO image of normal-phase domains in the intermediate state of indium in an external magnetic field $H_{\text {ext }}=30$ Oe at temperature $T=2.6 \mathrm{~K}$. The bright
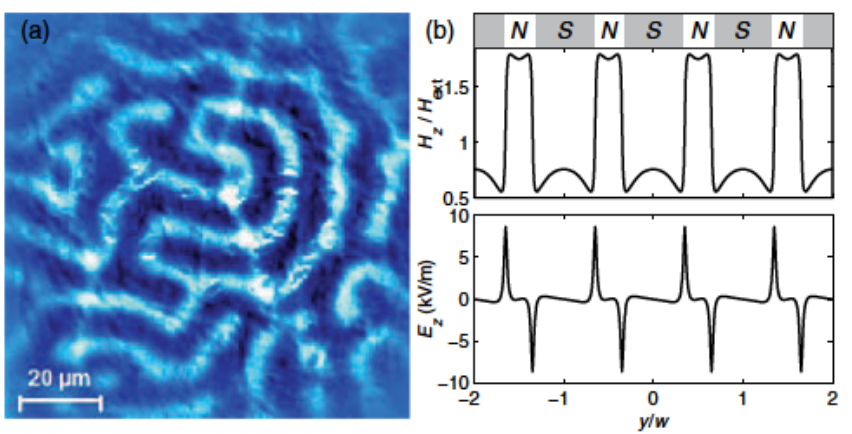

FIG. 2 (color online). (a) MO imaging of normal state domains in the intermediate state of indium. $H_{\text {ext }}=30 \mathrm{Oe}$ and $T=2.6 \mathrm{~K}$. (b) Theoretical profiles of the magnetic field and the electric field due to the flexomagnetoelectric effect, calculated in the molecular layer in a simplified geometry where $N$ and $S$ domain walls are flat and form a periodic structure aligned along the $x$ axis.

disordered lamella-shaped structures correspond to normalstate regions $(N)$, while superconducting areas $(S)$ appear dark, with an average period of the domain structures of the order of $10 \mu \mathrm{m}$. To theoretically estimate the electric field generated by the Bi:LuIG film in the organic molecular layer, we assume that $N$ and $S$ domains in the superconductor are along the $x$ direction and form a periodic structure with period $w$ in the $y$ direction [see Fig. 2(b)]. The screening supercurrents at the $N / S$ boundaries induce a magnetic field $\mathbf{H}=H_{y}(y, z) \hat{\mathbf{y}}+H_{z}(y, z) \hat{\mathbf{z}}$. As a consequence, the magnetization in the garnet film becomes a superposition of a large uniform in-plane magnetic moment $\mathbf{M}_{s}=M_{s}(\cos \theta \hat{\mathbf{x}}+\sin \theta \hat{\mathbf{y}})$ and a small nonuniform magnetization $\mathbf{M}=\chi_{m} \mathbf{H}$, where $\chi_{m}$ is the magnetic susceptibility of the garnet. According to Eq. (1) the induced electric polarization has the form

$$
\mathbf{P}(y, z)=\gamma \chi_{e} \chi_{m} M_{s} \sin \theta \frac{\partial}{\partial y} \mathbf{H}(y, z) .
$$

The strength of the flexomagnetoelectric effect in the garnet film strongly depends on the orientation of $\mathbf{M}_{s}$ and is maximal when $\mathbf{M}_{s}$ is perpendicular to $N / S$ domain walls. In the following we consider only the case where $\theta=\pi / 2$. For simplicity, we also assume that the $N / S$ domain walls are flat and that the magnetization $\mathbf{M}$ induced by the superconducting currents in the indium film has only one spatial component along the $z$ axis. Thus, in the superconducting regions $4 \pi M_{z}=-H_{\text {ext }}$, while in the normal regions $4 \pi M_{z}=H_{\text {ext }}\left(d_{S} / d_{N}\right)$, where $d_{N}=w\left(H_{\text {ext }} / H_{c}\right)$ and $d_{S}=w-d_{N}$ are the widths of the $N$ and $S$ domains, respectively, with $H_{c}$ being the critical field. Solving the Maxwell equations for the magnetic field induced by the superconductor in the $\mathrm{Bi}: \mathrm{LuIG}$ and choosing the origin of the coordinate system at the surface of the superconductor in the center of a $S$ domain, one finds

$$
\mathbf{H}=\mathbf{H}_{\mathrm{ext}}+\sum_{n=1}^{\infty} H_{n}(z)\left[\sin \left(q_{n} y\right) \hat{\mathbf{y}}+\cos \left(q_{n} y\right) \hat{\mathbf{z}}\right],
$$


with

$$
H_{n}(z)=-\frac{2 H_{\mathrm{ext}}}{q_{n} d_{N}}\left(1-e^{q_{n} D}\right) e^{q_{n} z} \sin \left(\frac{q_{n} d_{S}}{2}\right),
$$

where $q_{n}=2 \pi n / w$. The Fourier harmonics of the electric polarization in the garnet film have the amplitudes $P_{n}(z)=\gamma \chi_{e} \chi_{m} M_{s} q_{n} H_{n}(z)$. Finally, taking the finite width $d_{i}$ of the garnet film into account, we obtain for the electric field induced by the polarization $\mathbf{P}(y, z)$ in the molecular layer $(0<z<d)[23]$ :

$$
\mathbf{E}=\sum_{n=1}^{\infty} E_{n}(z)\left[\cos \left(q_{n} y\right) \hat{\mathbf{y}}+\sin \left(q_{n} y\right) \hat{\mathbf{z}}\right]
$$

with

$$
\begin{aligned}
E_{n}(z)= & \frac{4 \pi \gamma \chi_{e} \chi_{m} M_{s} H_{c}}{w}\left(1-e^{q_{n} D}\right)\left(1-e^{2 q_{n} d_{i}}\right) \\
& \times e^{q_{n}(2 d z)} \sin \left(q_{n} d_{S} / 2\right) .
\end{aligned}
$$

The profiles of the magnetic and electric fields in the molecular layer along the $y$ axis are shown in Fig. 2(b). The vertical component of the electric field is maximal close to each $N / S$ boundary due to the rotation of the in-plane magnetization [see Fig. 1(a)]. Taking the parameters relevant to our experimental conditions, $w=10 \mu \mathrm{m}, d_{S}=1.6 \mu \mathrm{m}$, $\chi_{e}=4 / 4 \pi$ [16], $\chi_{m}=0.11, \gamma=10^{8} \mathrm{erg}^{1 / 2} \mathrm{~cm}^{1 / 2} \mathrm{G}^{2}$, electric fields of the order of $10^{4} \mathrm{~V} / \mathrm{m}$ are expected. These field amplitudes can induce Stark shifts of the molecular resonances comparable to their linewidths.

To probe the electric field generated by the Bi: LuIG at a single molecule located in the organic layer, we modulate the amplitude of the external magnetic field in such a way that normal-phase domains undergo a reversible oscillatory in-plane motion. The molecule will therefore experience a temporally modulated local electric field due to the flexomagnetoelectric effect. Because of the large distribution in the pinning strengths of normal-phase domains in the indium layer, the amplitude of the magnetic field modulation depends on the probed area of the sample and was chosen to produce noticeable displacements of the domains [39]. As exemplified in Fig. 3(a), domain boundaries created at $H_{\text {ext }}=30 \mathrm{Oe}$ displayed micrometric displacements for a modulation amplitude of 2Oe. As shown in this figure, simultaneous MO and single-molecule fluorescence imaging on the same camera allows the selection of a single molecule (the circled bright spot) close to an oscillating domain (delimited by the dashed lines). The periodic variations of the molecule fluorescence intensity at the modulation frequency $1 \mathrm{~Hz}$ are displayed in Fig. 3(b). Here, the excitation laser frequency was tuned to the flank of the Lorentzian-shaped molecular line in order to maximize the sensitivity of the fluorescence signal to molecular spectral shifts. We checked to ensure that the modulation of the fluorescence signal disappears above the critical temperature of indium, i.e., when the magnetic field
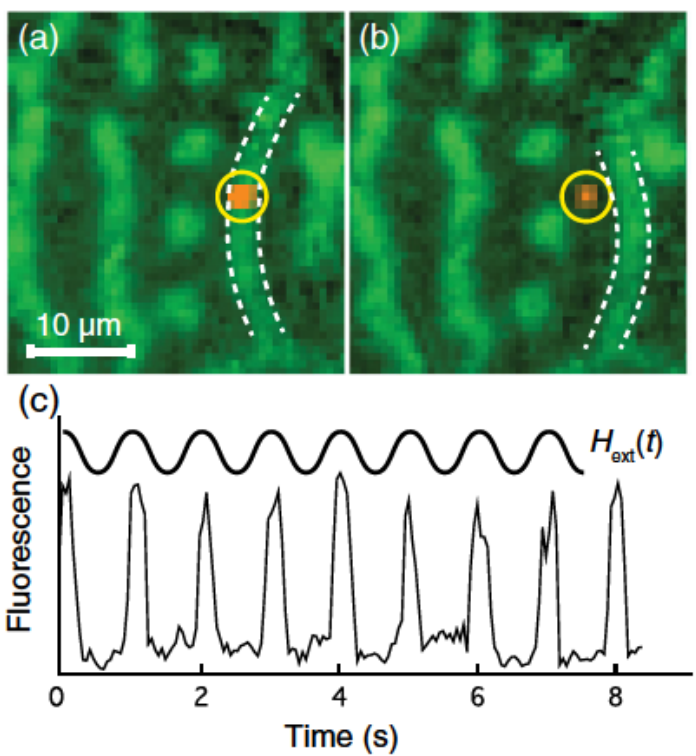

FIG. 3 (color online). Single molecule imaging with simulta neous MO imaging of normal phase domains in the indium film. $H_{\text {ext }}$ is modulated at $1 \mathrm{~Hz}$ with a modulation amplitude of $2 \mathrm{Oe}$ around $30 \mathrm{Oe} . T=2.6 \mathrm{~K}$. The laser frequency is fixed to the flank of the Lorentzian shaped molecular line. (a) $H_{\text {ext }}=29$ Oe. A single molecule (the circled bright spot) is chosen above a normal phase domain (delimited by the dashed lines). (b) Image of the same area at $H_{\text {ext }}=31$ Oe illustrating the motion of the domain boundaries. (c) Temporal evolution of the fluorescence intensity of that molecule probing the flexomagnetoelectric effect.

uniformly penetrates the indium layer. From the amplitude of the signal variations, we deduce that the molecule expresses a modulated Stark shift of the order of its linewidth $(50 \mathrm{MHz})$. Using the measurement of the linear Stark coefficient $0.8 \mathrm{GHz} /(\mathrm{MV} / \mathrm{m})$ for this molecule [23], we estimate a local electric field variation of $\sim 60 \mathrm{kV} / \mathrm{m}$. This value is compatible with the above theoretical estimations of the fields created by the flexomagnetoelectric effect. Evidence of this effect was found at various positions in the sample for more than 20 molecules which displayed fluorescence intensity variations upon moving $N / S$ boundaries. Estimation of the local electric field probed by eight of those molecules is presented in the Supplemental Material [23].

In order to rule out any origin of the signal variations other than molecular spectral shifts, we recorded the fluorescence signal under simultaneous modulation of the magnetic field and a periodic scan of the laser wavelength at the same frequency $\omega_{\text {mod. }}$. As illustrated in Fig. 4(a), if the signal variations are due to a spectral modulation of the molecular transition due to the flexomagnetoelectric effect, their amplitude should strongly depend on the relative phase $\varphi$ between the magnetic field modulation and the laser scan. Indeed, the fluorescence intensity of the molecule depends on the frequency detuning between the 
(a)
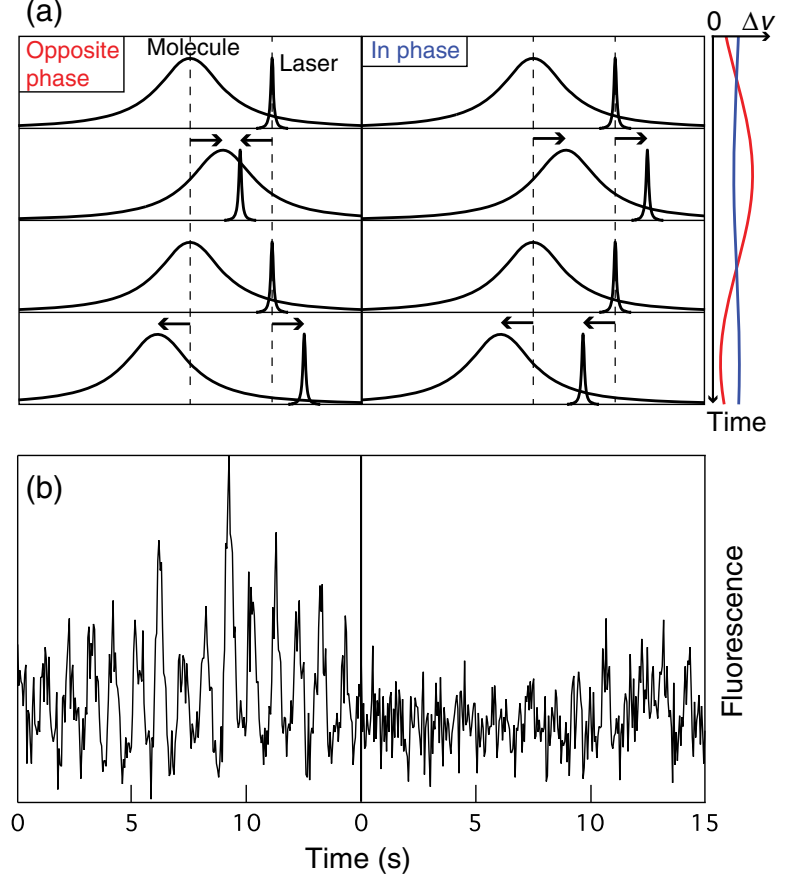

FIG. 4 (color online). Temporal evolution of the fluorescence signal of a molecule probing the flexomagnetoelectric effect, under synchronized modulations of the magnetic field and the laser wavelength. (a) Schematic drawing of the modulated laser molecule frequency detuning, in the cases of opposite phase ( $\varphi=\pi$, red curve) and in phase $(\varphi=0$, blue curve) modulations. (b) Temporal evolution of the fluorescence signal of a single molecule located close to an oscillating domain, in the two phase configurations. (Left) Opposite phase. (Right) In phase.

molecule resonance frequency and the laser frequency $\delta(t)=\delta_{0}+A_{L} \cos \left(\omega_{\bmod } t+\varphi\right)-A_{M} \cos \left(\omega_{\bmod } t\right)$, where $A_{L}$ is the amplitude of the laser scan and $A_{M}$ is the amplitude of the molecular Stark shift. When $\varphi=\pi$, a large modulation of the laser-molecule detuning (with an amplitude $A_{M}+A_{L}$ ) and, therefore, of the fluorescence signal is obtained. In the case of $\varphi=0$, a weak modulation of the laser-molecule detuning (with an amplitude $\left|A_{M}-A_{L}\right|$ ) leads to small variations in the fluorescence signal. This behavior is clearly observed in Fig. 4(b), where we compare the temporal evolution of the fluorescence signal of a single molecule located close to an oscillating domain in the two phase configurations. This method also allows a determination of the Stark shift amplitude $A_{M}$ by adjusting the laser scan amplitude $A_{L}$ to the value corresponding to the zero fluorescence signal for $\varphi=0$ (in this situation, $A_{M}=A_{L}$ ). For this molecule, the linear Stark coefficient is $1 \mathrm{GHz} /(\mathrm{MV} / \mathrm{m})$ and fluorescence modulations vanish for $A_{L}=40 \mathrm{MHz}$, yielding an estimation of the local electric field variations of $\sim 40 \mathrm{kV} / \mathrm{m}$.

In conclusion, we used single-molecule fluorescence spectroscopy to probe local electric fields generated by the flexomagnetoelectric effect in a Bi:LuIG film. This effect was produced with the nonuniform magnetic fields at the $N / S$ domain boundaries of an indium layer. It was evidenced by the Stark effect of eight single molecules which revealed local electric field variations up to $60 \mathrm{kV} / \mathrm{m}$, in accordance with the theoretical estimations. The disparity in the measured electric fields can be attributed to the various orientations of $N / S$ domain walls with respect to the in-plane magnetization in the Bi:LuIG film, and to the random orientations of the probe molecules in the organic layer. This observation paves the way for the use of ultrasensitive nanometric probes such as single fluorescent molecules to directly investigate local electric fields in the condensed matter and to probe nanomechanical motions of charged oscillators [40].

We gratefully acknowledge our stimulating and fruitful discussions with A.P. Pyatakov, L.S. Uspenskaya, V. Vlasko-Vlasov, and D. Roditchev. This work was supported by the ANR (Project ElectroVORTEX), the Institut Universitaire de France, the French ministry of Education and Research, and the European NanoSC COST Action MP1201.

[1] S. W. Cheong and M. Mostovoy, Nat. Mater. 6, 13 (2007).

[2] W. Eerenstein, N. D. Mathur, and J. F. Scott, Nature (London) 442, 759 (2006).

[3] S. Zhang, Electric Field Control of Magnetization and Electronic Transport in Ferromagnetic/Ferroelectric Heterostructures, Springer Theses Vol. XVI (Springer, New York, 2014).

[4] D. Khomskii, Physics 2, 20 (2009).

[5] V. E. Koronovskyy, S. M. Ryabchenko, and V. F. Kovalenko, Phys. Rev. B 71, 172402 (2005).

[6] B. B. Krichevtsov, V. V. Pavlov, and R. V. Pisarev, JETP Lett. 49, 535 (1989); B. B. Krichevtsov, V. V. Pavlov, R. V. Pisarev, and A. G. Selitsky, Ferroelectrics 161, 65 (1994).

[7] A. P. Pyatakov and A. K. Zvezdin, Phys. Usp. 55, 557 (2012).

[8] H. Katsura, N. Nagaosa, and A. V. Balatsky, Phys. Rev. Lett. 95, 057205 (2005).

[9] M. Mostovoy, Phys. Rev. Lett. 96, 067601 (2006).

[10] V. G. Bar'yakhtar, V. A. L'vov, and D. A. Yablonskii, JETP Lett. 37, 673 (1983).

[11] I. Dzyaloshinskii, Europhys. Lett. 83, 67001 (2008).

[12] A. S. Logginov, G. A. Meshkov, A. V. Nikolaev, and A. P. Pyatakov, JETP Lett. 86, 115 (2007).

[13] A. S. Logginov, G. A. Meshkov, A. V. Nikolaev, E. P. Nikolaeva, A. P. Pyatakov, and A. K. Zvezdin, Appl. Phys. Lett. 93, 182510 (2008).

[14] A. P. Pyatakov, D. E. Sechin, and A. S. Sergeev, Europhys. Lett. 93, 17001 (2011).

[15] A. P. Pyatakov, G. A. Meshkov, and A. K. Zvezdin, J. Magn. Magn. Mater. 324, 3551 (2012).

[16] A. F. Kabychenkov, F. V. Lisovskii, and E. G. Mansvetova, JETP Lett. 97, 265 (2013).

[17] W. E. Moerner and L. Kador, Phys. Rev. Lett. 62, 2535 (1989); M. Orrit and J. Bernard, Phys. Rev. Lett. 65, 2716 (1990). 
[18] Single Molecule Optical Detection, Imaging, and Spectros copy, edited by Th. Basche, W. E. Moerner, M. Orrit, and U. P. Wild (Wiley VCH, Munich, 1997); M. Orrit, J. Chem. Phys. 117, 10938 (2002).

[19] Ph. Tamarat, A. Maali, B. Lounis, and M. Orrit, J. Phys. Chem. A 104, 1 (2000).

[20] C. Brunel, Ph. Tamarat, B. Lounis, J. C. Woehl, and M. Orrit, J. Phys. Chem. A 103, 2429 (1999).

[21] M. Bauer and L. Kador, Chem. Phys. Lett. 407, 450 (2005).

[22] A. M. Boiron, Ph. Tamarat, B. Lounis, R. Brown, and M. Orrit, Chem. Phys. 247, 119 (1999).

[23] See Supplemental Material at http://link.aps.org/ supplemental/10.1103/PhysRevLett.115.027601, which in cludes Refs. [12, 16, 20, 24 32, 37], for more experimental details and the estimation of the electric fields.

[24] O. Labeau, Ph. Tamarat, H. Courtois, G. S. Agarwal, and B. Lounis, Phys. Rev. Lett. 98, 143003 (2007).

[25] R. V. Pisarev, I. G. Sinii, N. N. Kolpakova, and Y. M. Yakovlev, Sov. Phys. JETP 33, 1175 (1971).

[26] G. A. Smolensky, R. V. Pisarev, I. G. Sinii, and N. N. Kolpakova, J. Phys. Colloq. C1 32, c1 1048 (1971).

[27] L. E. Helseth, A. G. Solovyev, R. W. Hansen, E. I. Il'yashenko, M. Baziljevich, and T. H. Johansen, Phys. Rev. B 66, 064405 (2002); P. Hansen, C. P. Klages, J. Schuldt, and K. Witter, Phys. Rev. B 31, 5858 (1985).

[28] J. I. Vestgården, D. V. Shantsev, A. A. F. Olsen, Y. M. Galperin, V. V. Yurchenko, P. E. Goa, and T. H. Johansen, Phys. Rev. Lett. 98, 117002 (2007).

[29] L.D. Landau and E. M. Lifshitz, Electrodynamics of Continuous Media (Pergamon Press, Oxford, 1984).
[30] L. E. Helseth, R. W. Hansen, E. I. Il'yashenko, M. Baziljevich, and T. H. Johansen, Phys. Rev. B 64, 174406 (2001).

[31] R. P. Huebener, Magnetic Flux Structures of Superconduc tors (Springer Verlag, Berlin, 2001).

[32] V. Jeudy, C. Gourdon, and T. Okada, Phys. Rev. Lett. 92, 147001 (2004); A. Cebers, C. Gourdon, V. Jeudy, and T. Okada, Phys. Rev. B 72, 014513 (2005); C. Gourdon, V. Jeudy, and A. Cebers, Phys. Rev. Lett. 96, 087002 (2006).

[33] L. A. Dorosinskii, M. V. Indenbom, V. I. Nikitenko, Yu. A. Ossip'yan, A. A. Polyanskii, and V. K. Vlasko Vlasov, Physica (Amsterdam) 203C, 149 (1992).

[34] A. A. Polyanskii, X. Y. Cai, D. M. Feldmann, and D. C. Larbalestier, in Nano Crystalline and Thin Film Magnetic Oxides, NATO Science Partnership Subseries 3, High Technology Vol. 72, edited by I. Nedkov and M. Ausloos (Kluwer Academic Publishers, Dordrecht, 1999), p. 353.

[35] A. A. Polyanskii, V. Beilin, D. M. Feldmann, M. Roth, E. Hellstrom, and D. Larbalestier, in Magneto Optical Imag ing, NATO Science Series II Vol. 142, edited by T. H. Johansen and D. V. Shantsev (Kluwer Academic Publishers, Dordrecht, 2004), p. 19.

[36] A. A. Polyanskii, Russ. Phys. J. 56, 1007 (2014).

[37] P. E. Goa, H. Hauglin, A. A. F. Olsen, M. Baziljevich, and T. H. Johansen, Rev. Sci. Instrum. 74, 141 (2003).

[38] Y. Tsuchiya, Y. Nakajima, and T. Tamegai, Physica (Amsterdam) 470C, 1123 (2010).

[39] J. Ge, J. Gutierrez, B. Raes, J. Cuppens, and V. V. Moshchalkov, New J. Phys. 15, 033013 (2013).

[40] V. Puller, B. Lounis, and F. Pistolesi, Phys. Rev. Lett. 110, 125501 (2013). 